\title{
miR-141 Inhibits Proliferation and Migration of Colorectal Cancer SW480 Cells
}

\author{
ZHI H. LONG ${ }^{1,2}$, ZHI G. BAI ${ }^{1}$, JIAN N. SONG $^{1}$, ZHI ZHENG ${ }^{1}$, JUN LI $^{1}$, JUN ZHANG $^{1}$, \\ JUN CAI ${ }^{1}$, HONG W. YAO ${ }^{1}$, JIN WANG ${ }^{1}$, YING C. YANG ${ }^{1}$, JIE YIN ${ }^{1}$ and ZHONG T. ZHANG ${ }^{1}$ \\ ${ }^{1}$ Department of General Surgery, Beijing Friendship Hospital, Capital Medical University, \\ Beijing Key Laboratory of Cancer Invasion and Metastasis Research \& National Clinical Research \\ Center for Digestive Diseases, Xi-Cheng District, Beijing, P.R. China; \\ ${ }^{2}$ School of Rehabilitation, Capital Medical University, Department of General Surgery, \\ Beijing Bo'ai Hospital, China Rehabilitation Research Center, Beijing, P.R. China
}

\begin{abstract}
Background: This study was designed to determine the molecular function of miR-141 and the underlying mechanisms in colorectal cancer (CRC). Materials and Methods: SW480 cells in which miR-141 was up- or down-regulated were established. Reverse transcription, quantitative polymerase chain reaction and Western blotting were used to examine the microRNA and protein expression. Cell-cycle progression was analyzed by flow cytometry. Proliferation marker Ki-67 was evaluated by immunofluorescence. Transwell assay was conducted to determine the migration rates of cells. Subcutaneous xenograft models were used to examine the effect of miR-141 on tumorigenicity. Human mitogen-activated protein kinase $(M A P K)$ and receptor tyrosine kinase (RTK) pathway phosphorylation array assays were used to interrogate MAPK and RTK pathway activation. Results: miR-141 directly targeted zinc finger E-box-binding homeobox $1 / 2$ (ZEB 1/2). We first determined the expression levels of ZEBI and ZEB2 in miR-141-expressing cells and miR-141knockdown cells and found that inhibition of miR-141 significantly increased the expression of ZEB2. In vitro study revealed that miR-141 overexpression inhibited the expression of Ki-67. Furthermore, overexpression of miR-141 led to a significant reduction in the proliferation of SW480 cells via induction of cell-cycle arrest at the $G_{1}$ stage. In
\end{abstract}

Correspondence to: Professor Zhong T. Zhang, and Dr Jie Yin, Department of General Surgery, Beijing Friendship Hospital, Capital Medical University, Beijing Key Laboratory of Cancer Invasion and Metastasis Research \& National Clinical Research Center for Digestive Diseases, 95 Yong-an Road, Xi-Cheng District, Beijing 100050, P.R. China. E-mail: Zhong T. Zhang: zhangzht@medmail.com.cn and Jie Yin: yj232325@sina.com

Key Words: miR-141, colorectal cancer (CRC), microRNA, metastasis. contrast, inhibition of miR-141 markedly promoted the proliferation of $\mathrm{SW480}$ cells by promoting cell-cycle progression. Moreover, overexpression of miR-141 significantly inhibited SW480 cell migration in vitro. In addition, overexpression of miR-141 significantly reduced tumor size and weight, and inhibited the growth of SW480 cell-derived tumor in nude mice. Notably, overexpression of miR-141 also suppressed the liver metastasis of SW480 cells in nude mice. Using RTK and MAPK arrays, we found increased phosphorylation of hepatocyte growth factor receptor (HGFR/c-MET) following inhibition of $m i R-141$, but phosphorylation of P53, AKT, ERK1/2, P38 and mTOR, etc., in SW480 cells was not affected by miR-141. Conclusion: Our results suggest that miR-141 functions as a tumor suppressor through ZEB2 and HGFR in CRC cells.

Colorectal cancer (CRC) is a common gastrointestinal malignancy and is the fourth most common cancer and the fifth most common cause of cancer-related death in China, with most patients diagnosed during the middle to late stages $(1,2)$. Distant metastasis is the major cause of death in patients with CRC. Liver metastases occur in approximately $15 \%$ of newly diagnosed patients (3). Treatment of CRC with liver metastases has become an international research hotspot in recent years. However, there remains a shortage of effective treatments for patients with metastatic CRC. Consequently, a better understanding of the molecular mechanisms underlying liver metastasis is required to promote the development of effective therapeutic strategies for patients with metastatic CRC. The development of CRC liver metastases is a complex process of multiple factors and multiple steps. There are a large number of molecules involved in the regulatory network. Our previous research found that serum $m i R 141$ was highly expressed in a patient with colorectal cancer and liver metastases. It was also found that the expression of zinc finger E-box-binding homeobox 
1 (ZEB1), which was one of the target proteins of miR141, had a significant correlation with the expression of Ecadherin, the biomarker of epithelial-mesenchymal transition (EMT) in metastatic tumor tissue (4).

MicroRNAs are a class of small, endogenous, non-coding RNAs that modify the expression of protein-coding genes by binding the sequence to the 3 '-untranslated region (3'-UTR) of target mRNA, leading to the repression of translation, or to degradation of the mRNA. MicroRNAs play critical roles in the initiation, development and metastasis of cancer by regulating their target genes (5-7). MicroRNA-141 ( $m i R$ $141)$ is a common cancer-associated miRNA. It has been reported as a tumor suppressor in some malignancies, such as gastric cancer, pancreatic cancer, breast cancer, renal cell carcinoma and hepatocellular carcinoma. However, the molecular function of miR-141 and its underlying mechanisms in CRC are still unclear.

In the present study, we evaluated the molecular function of miR-141 and underlying mechanisms in a panel of CRC cell lines. SW480 cells in which $m i R-141$ was up- or downregulated were established. Reverse transcription-quantitative polymerase chain reaction (RT-qPCR), western blot, receptor tyrosine kinase (RTK) and mitogen-activated protein kinase (MAPK) array was used in the study.

\section{Materials and Methods}

Cell lines and cell culture. Human colorectal carcinoma cell lines SW480 and HCT116 were purchased from the Institute of Biochemistry and Cell Biology, Chinese Academy of Sciences (Shanghai, PR China). All cells were cultured in RPMI-1640 medium (Gibco, Carlsbad, CA, USA) supplemented with $10 \%$ fetal bovine serum (FBS; Gibco) and $1 \%$ penicillin-streptomycin, and grown in a humidified incubator at $37^{\circ} \mathrm{C}$ with $5 \% \mathrm{CO}_{2}$.

Establishment and of culture miR-141 under-and overexpressing cells. The lentivectors for expression of $m i R-141$ and antisense $m i R$ 141 were purchased from the GenePharma Company (Shanghai, PR China). Lentivectors and packaging vectors were co-transfected into HEK-293T cells (the Institute of Biochemistry and Cell Biology, Chinese Academy of Sciences, Shanghai, P.R. China) with Lipofectamine 2000 (Invitrogen, Carlsbad, CA, USA) according to the manufacturer's protocol. After $48 \mathrm{~h}$ transfection, the culture supernatant was collected and centrifuged at $4,000 \times g$ for $10 \mathrm{~min}$. The supernatant was filtered and centrifuged to harvest the high-titer lentivirus containing $m i R-141$ or antisense miR-141. After determination of the virus titer, the mixture of an appropriate volume of virus stock solution and cell culture medium were added to SW480 and HCT116 cells. After $72 \mathrm{~h}$ incubation, the cells were selected using puromycin $(5 \mu \mathrm{g} / \mathrm{ml})$ for 21 days to establish stably transfected cell miR-141-overexpressing and miR-141-inhibited (miR-141-in) cell lines. Successfully transfected cells were confirmed by their expression of ZEB1 and ZEB2 using western blot.

Western blot. Total cellular proteins were extracted using cell lysis buffer [20 mM Tris/HCl, $150 \mathrm{mM} \mathrm{NaCl}, 1 \%$ Nonidet P-40, $0.1 \%$ sodium dodecyl sulfate (SDS) and protease inhibitor cocktail, $\mathrm{pH}$ 7.5]. The protein concentrations of the lysates were determined according to the bicinchoninic acid method using a protein assay kit (Pierce, Rockford, IL, USA).Samples containing $50 \mu \mathrm{g}$ of protein were separated by $12 \%$ SDS-polyacrylamide gel electrophoresis. After electrophoresis, the proteins were transferred to a polyvinylidene difluoride membrane (Millipore, Billerica, MA, USA) for western blot analysis. Primary antibodies against ZEB1 (diluted 1:500; Santa Cruz Biotechnology, Santa Cruz, CA, USA), ZEB2 (diluted 1:500; Santa Cruz Biotechnology), and glyceraldehyde 3-phosphate dehydrogenase (GAPDH, diluted 1:10,000; KangChen, Shanghai, PR China) were used. The blotted membranes were washed and incubated with secondary antibodies conjugated to horseradish peroxidase. Immunoreactive proteins were detected by enhanced chemiluminescence (ECL; Applygen Technologies Inc., Beijing, China). The relative density of the protein bands was quantitatively determined with the ImageJ software (National Institutes of Health, Bethesda, MD, USA).

Cell-cycle analysis. Cells were harvested and washed twice with cold phosphate-buffered saline (PBS), and then fixed in $70 \%$ icecold ethanol. The fixed cells were treated with RNase A (SigmaAldrich, St. Louis, MO, USA) for $20 \mathrm{~min}$, followed by incubation with propidium iodide (PI, Beyotime, Shanghai, China) for $30 \mathrm{~min}$. Cell-cycle analysis was performed using a FACS Calibur (BD Biosciences, USA). FlowJo software was used to determine the percentage of cells in $\mathrm{G}_{0} / \mathrm{G}_{1}, \mathrm{~S}$ and $\mathrm{G}_{2} / \mathrm{M}$ phases.

Immunofluorescence. Cells were fixed in $4 \%$ paraformaldehyde for $20 \mathrm{~min}$ at room temperature (RT), and then permeabilized with $0.1 \%$ Triton X-100 in PBS for 15 min at RT. The cells were then blocked for $1 \mathrm{~h}$ at RT. Next, the cells were incubated with primary antibody $\mathrm{Ki} 67$ (1:200, Abcam, Cambridge, UK) overnight at $4^{\circ} \mathrm{C}$. After being rinsed in PBS, the cells were incubated in redfluorescent Alexa Fluor 594 goat anti-rabbit IgG antibody (1:200; Invitrogen, Carlsbad, CA, USA) for $1 \mathrm{~h}$ in the dark. Cells were washed and then incubated for 10 min with 4'-6-diamidino-2phenylindole (DAPI; Beyotime, Shanghai, P.R. China) to monitor the nuclei. Fluorescent staining was visualized using a fluorescence microscope.

Transwell assay. Twenty-four-well transwell plates $(8-\mu \mathrm{m}$ pore size; Corning, Cambridge, MA, USA) were used to measure the migratory ability of each cell line. Briefly, $1 \times 10^{5}$ cells were resuspended in RPMI-1640 medium free of FBS and seeded in the upper chamber. Medium containing 20\% FBS was supplemented into the lower compartment. After incubation for $24 \mathrm{~h}$ in an incubator, the cells were fixed with $4 \%$ paraformaldehyde and stained with DAPI. Cells remaining on the upper surface of the filter membrane (nonmigrating) were removed with the cotton stick. The migrating cells on the lower surface were captured by a fluorescence microscope and counted at least 10 random fields per chamber.

Animal experiments. 6-Week-old male BALB/c nude mice were purchased from the Academy of Military Medical Sciences (Beijing, PR China) and maintained under controlled conditions with a 12-h light-dark cycle. All protocols related to animal management were performed in accordance with the guidelines of Ethics Committee of the Capital Medical University.

For the tumorigenicity study, $1 \times 10^{6} m i R-141$ or $m i R-141$-in cells were re-suspended in RPMI-1640 medium and subcutaneously 


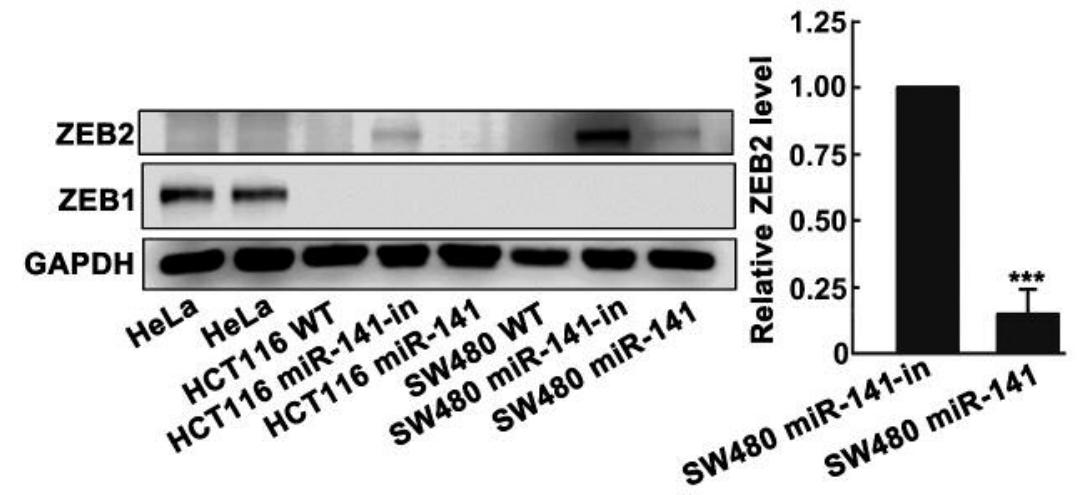

Figure 1. The zinc finger E-box-binding homeobox 1 (ZEB1) and ZEB2 protein levels in miR-14-overexpressing (miR-141) and-inhibited (miR-141in) colorectal cancer cells were analyzed by western blot.The relative density of the ZEB2 band was normalized to that of glyceraldehyde 3-phosphate dehydrogenase $(G A P D H)$. The values are expressed as the means $\pm S D$ s of four independent experiments. The values are expressed as a ratio of the band intensities of the miR-141-in cells. ***Significantly different at $p<0.001$ compared with miR-141-in cells.

injected into the left and right armpits of nude mice. In all, six mice (12 tumor sites) were injected. Nine days post-injection, tumor size was measured every 3 days, and tumor volumes were calculated using the formula, tumor volume $\left(\mathrm{mm}^{3}\right)=0.5 \times$ length $(\mathrm{mm}) \times$ width $(\mathrm{mm}) \times$ height $(\mathrm{mm})$. On the 21 st day post-injection, the mice were sacrificed and the weight of tumor was measured.

For the metastasis study, $1 \times 10^{6} \mathrm{miR}-141$ or $m i R-141$-in cells were injected into groups of six nude mice via the spleen. After 60 days post-injection, the mice were sacrificed and the recipient livers were dissected. The hepatic metastatic nodules were then counted.

Antibody arrays. RayBio ${ }^{\circledR}$ C-Series Human and Mouse MAPK Pathway Phosphorylation Array $\mathrm{C} 1$ and RayBio ${ }^{\circledR} \mathrm{C}$-Series Human RTK Phosphorylation Antibody Array C1 (RayBiotech, Norcross, GA, USA) were used to detect the level of phosphorylation of 17 human proteins and 71 different human receptor tyrosine kinases (RTKs) in cell lysates according to the manufacturer's protocol. Briefly, miR-141-, and miR-141-transfected SW480 cells were lysed, and the total proteins were purified. Then, $500 \mu \mathrm{g}$ of these proteins were added to a blocked membrane and incubated at $4^{\circ} \mathrm{C}$. The membrane was then washed and incubated with the biotinylated antibody cocktail. The signals were scanned using ImageQuant LAS4000 (GE, Pittsburgh, PA, USA) and analyzed using AAHMAPK-1 or AAH-PRTK-1 software (RayBiotech, Norcross, GA, USA). The median signal score was averaged across the duplicates on each array.

Data analysis. The data are expressed as the mean \pm standard deviation (SD). The experimental data were analyzed with the Statistical Product and Service Solutions (SPSS) 19.0 software (IBM Corp., Armonk, NY, USA). Statistical significance was examined using Student's two tailed $t$-test. A value of $p<0.05$ was considered statistically significant.

\section{Results}

Inhibition of miR-141 enhanced CRC cell proliferation and migration in vitro. In order to investigate the effects of $m i R$ 141 on cell proliferation and migration of CRC, we used two colorectal cell lines SW480 and HCT116 to establish $m i R$ 141 -overexpressing cells and $m i R$ - 141 -in cells. ZEB1 and ZEB2 are directly targeted by $m i R-141$, hence the expression of ZEB1/2 was measured by western blot to evaluate the expression level of $m i R-141$ in $m i R-141$ cells and $m i R-141$ in cells. As shown in Figure 1, expression of ZEB1 was not detected in SW480 cells nor in HCT116 cells. The expression of ZEB2 in SW480 cells was significantly increased in miR-141-in cells compared to miR-141overexpressing cells $(p<0.001)$. These results showed $m i R$ 141 overexpression reduced ZEB2 protein expression, while miR-141 knockdown increased its expression in SW480 cells. We therefore used the SW480 miR-141 and miR-141in cells in subsequent experiments.

Flow cytometry was used to investigate the effects of $m i R$ 141 on the cell cycle. The data showed that there was a dramatic decrease in the percentage of cells in the $G_{1} / G_{0}$ phase $(26.45 \%)$ in SW480 $m i R$ - 141 -in cells compared with SW480 miR-141 cells $(34.46 \%, p<0.01)$. In contrast, there was a significant increase in the percentage of cells in the $\mathrm{S}$ phase $(54.87 \%)$ and $\mathrm{S}+\mathrm{G}_{2} / \mathrm{M}$ phase $(73.55 \%)$ in $\mathrm{miR} 141$-in SW480 cells compared to miR-141-overexpressing cells (35.89\% and $65.54 \%$, respectively, $p<0.001$ and $p<0.05$, respectively) (Figure 2A and B). Similarly, the results shown in Figure $2 \mathrm{C}$ indicate that inhibition of $m i R-141$ dramatically increased the Ki67 staining of SW480 cells.

We next determined the change of migratory ability in miR-141-transfected CRC cells through Transwell assay. We found inhibition of $m i R-141$ in SW480 cells significantly promoted cell migration. The number of migrating antisense $m i R-141$ cells was notably increased compared with the $m i R$ 141 overexpressing cells $(p<0.001$, Figure 2D). Together, these findings suggest $m i R-141$ inhibits cell proliferation and migration of CRC cells. 

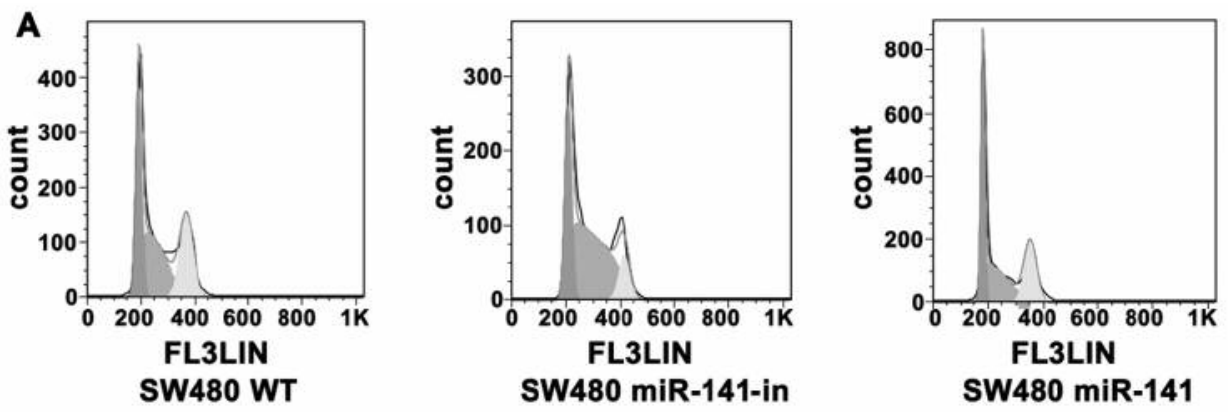

B

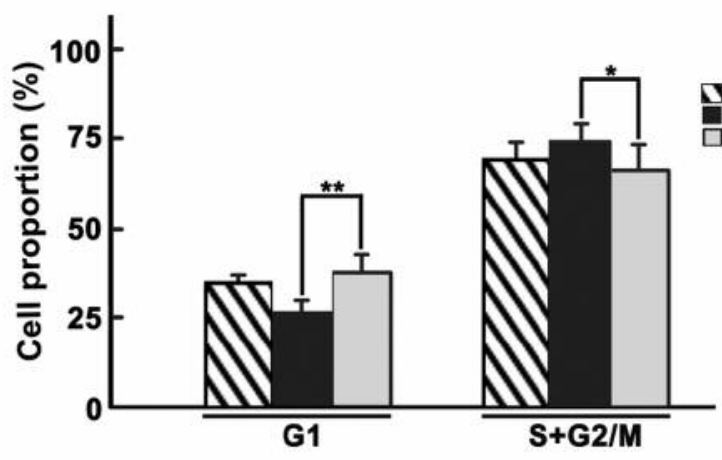

C
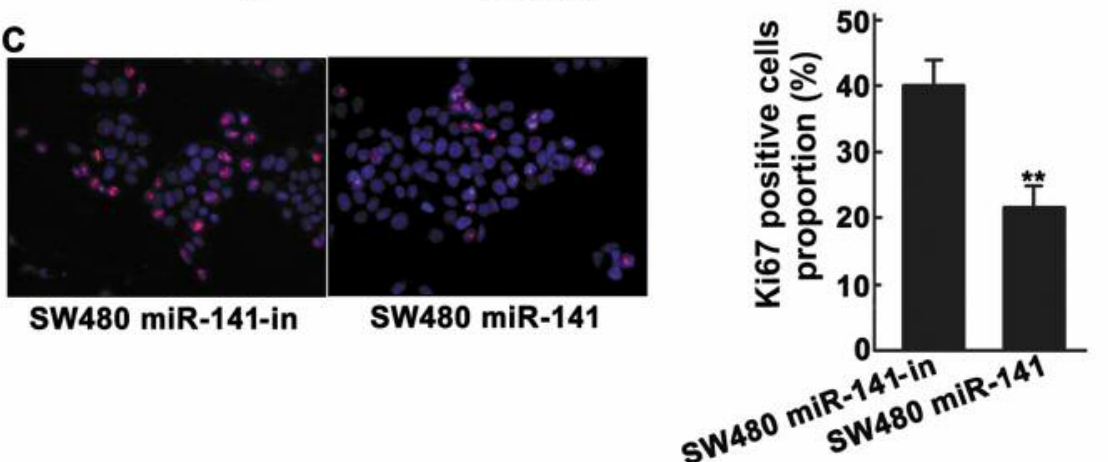

D
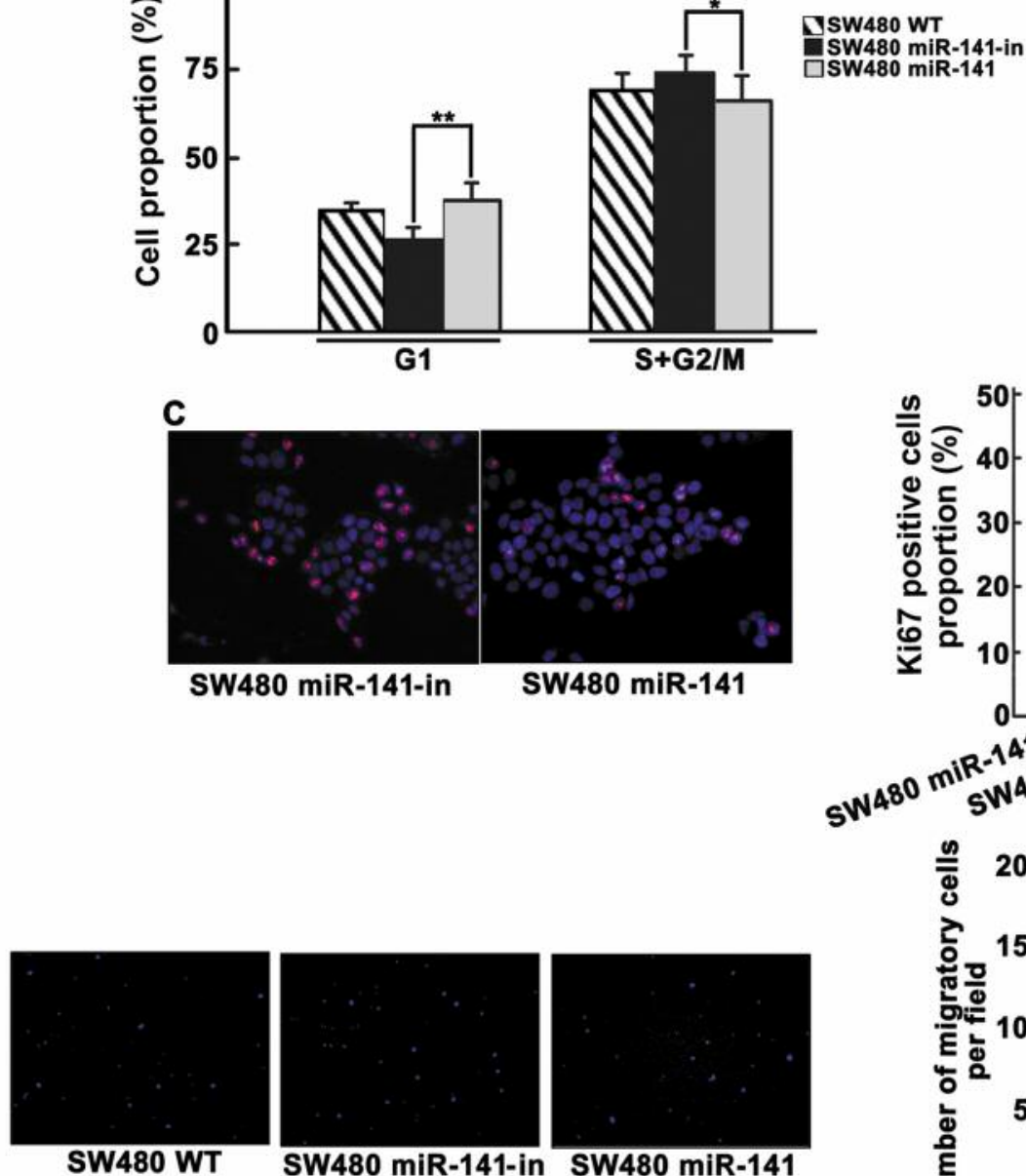

SW480 WT SW480 miR-141-in SW480 miR-141

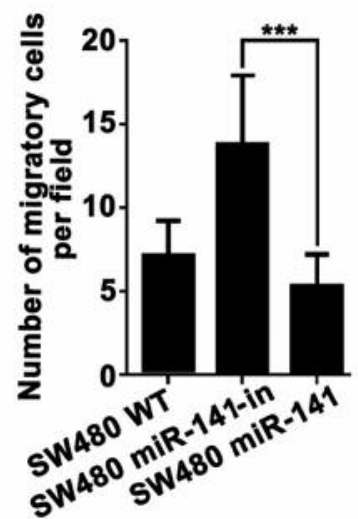

Figure 2. Expression of miR-141 inhibited proliferation and migration of SW480 cells. A and B: Flow cytometric analyses of miR-14-overexpressing (miR-141) and-inhibited (miR-141-in) SW480 cells.The percentage of $G_{0} / G_{1}$ phase cells was decreased, and the percentage of $S$ and $G_{2} / M$ phase cells was increased in miR-141-in cells. Each histogram bar in $(B)$ represents the mean $\pm S D s$ of four independent experiments. Differences are significant at $* p<0.05$ and $* * p<0.01$. C: Left: Cell growth was monitored by Ki67 immunofluorescent staining (red), and the nuclei were stained with 4,6-diamino-2-phenyl indole (DAPI) (blue). Right: Quantitative analysis of Ki67+ cells (at least 50 cells per group per experiment) from six randomly fields in each individual experiment. The values are expressed as the means \pm SDs of four independent experiments. **Significantly different at $p<0.01$ compared to miR-141-in cells. D: Down-regulation of miR-141 promoted the migration of SW480 cells in the transwell assay. Left: Representative views of migratory cells on the membrane. The nuclei of migratory cells were stained with DAPI (blue). Right: Quantitative data are represented as mean \pm SDs ( $n=4$ for each group). $* * *$ Significantly different at $p<0.001$. WT: Wild-type. 


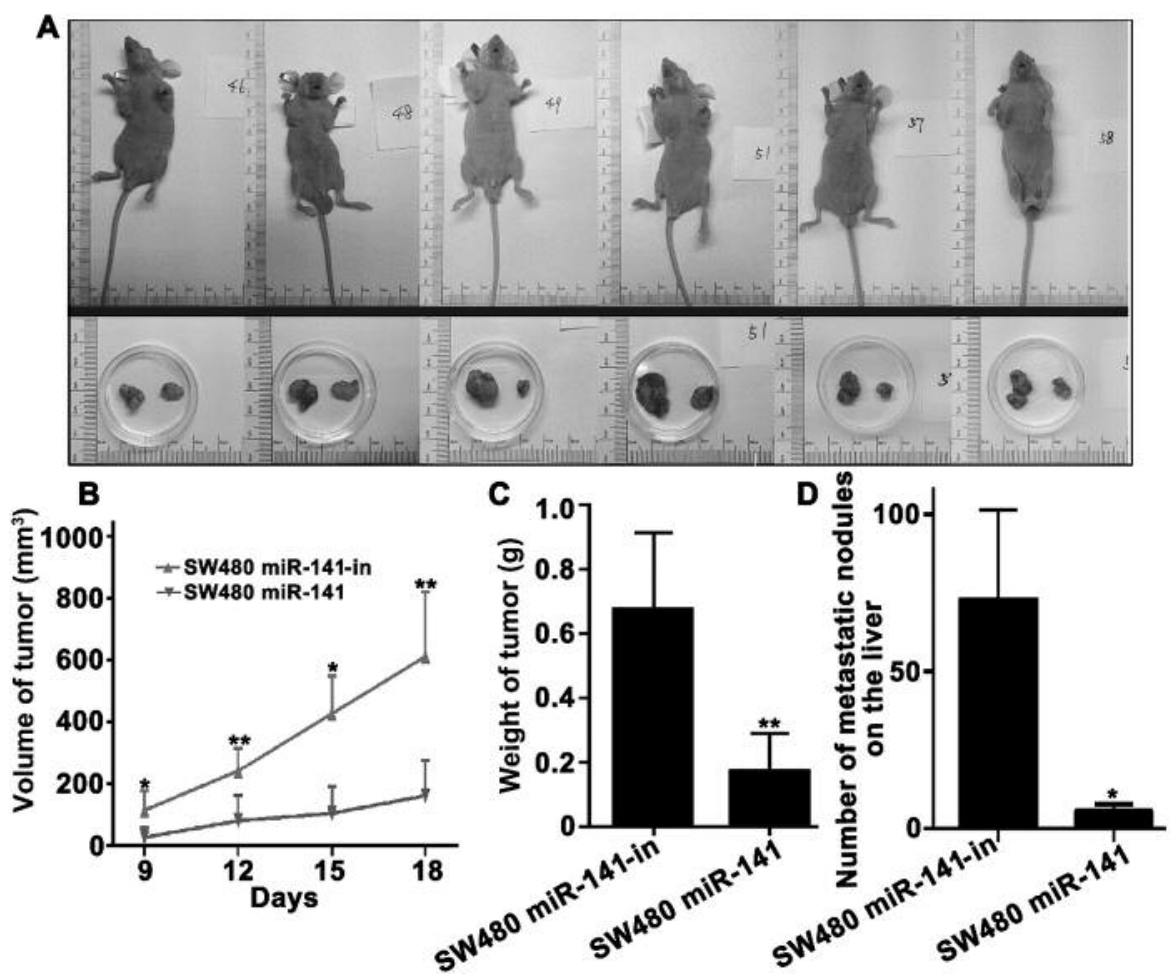

Figure 3. miR-141 reduced tumor growth and hepatic metastasis of SW480 cells in vivo. A total of $1 \times 10^{6}$ miR-141-overexpressing (miR-141) or -inhibited (miR-141-in) cells were injected subcutaneously into the left and right armpits of nude mice ( $n=6)(A)$. Nine days after injection, tumor volume measurement began and was performed every 3 days $(B)$. After 18 days, the tumor weight was determined $(C)$. D: A total of $1 \times 10^{6}$ miR141 and miR-141-in cells were injected into the spleens of nude mice $(n=6)$. After 60 days, metastatic nodules on the liver surface were counted. Data are represented as mean $\pm S D\left(n=6\right.$ for each group). Significantly different at $* p<0.05$, ${ }^{*} p<0.01$.

Knockdown of miR-141 promotes colorectal tumor growth and hepatic metastasis in vivo. To further investigate the in vivo effect of $m i R-141$ on tumor formation and metastasis, SW480 cells stably transfected with either miR-141 or antisense miR-141, and were subcutaneously and intrasplenically injected into nude mice. For the tumorigenicity study, $m i R-141$ SW480 cells and $m i R-141$-in SW480 cells were subcutaneously injected into the left and right armpits of nude mice. Nine days post-injection, the growth curves of tumor from miR-141-overexpressing versus antisense miR-141-transfected cells were compared (Figure $3 \mathrm{~B})$. As shown, the tumor volume in tumors with antisense $m i R-141$ was consistently higher than in tumors with miR141 overexpression, in particular, at day 18 after injection $(p<0.01)$. Similarly, the average weight of the tumors derived from $m i R$ - 141 -overexpressing cells at the end of the experiment was significantly reduced as compared to that of the tumors from $m i R-141$-in cells $(p<0.01$, Figure 3C).

In the metastasis study, fewer hepatic metastatic nodules were found in mice with cells that overexpressed miR-141 compared to cells in which miR-141 was down-regulated $(p<0.05$, Figure 3D). In coordination with the in vitro study, overexpression of $m i R-141$ was found to inhibit colorectal tumor progression and hepatic metastasis.

MAPK and RTK activity in the miR-141 and miR-141-in $S W 480$ cells. In order to elucidate the overall effects of miR141 on CRC cell signaling, MAPK and RTK Phosphorylation Antibody Arrays were applied to detect the changes in phosphorylation of signaling proteins in SW480 cells. We assessed phosphorylating of 17 proteins of the MAPK pathway in miR-141 cells compared to miR-141-in cells. As shown in Figure 4A, based on the search criterion for differential expression, which was a $>1.5$-fold induction or repression, signaling intensity analysis did not detect differential expression between the groups. The results suggest that the phosphorylation of P53, AKT, extracellularregulated protein kinases $1 / 2$ (ERK1/2), P38 and mammalian target of rapamycin (mTOR), in SW480 cells was not affected by $m i R-141$. We next examined the phosphorylation of 71 human RTKs. Increased phosphorylation of hepatocyte growth factor receptor (HGFR, also known as c-MET) was 


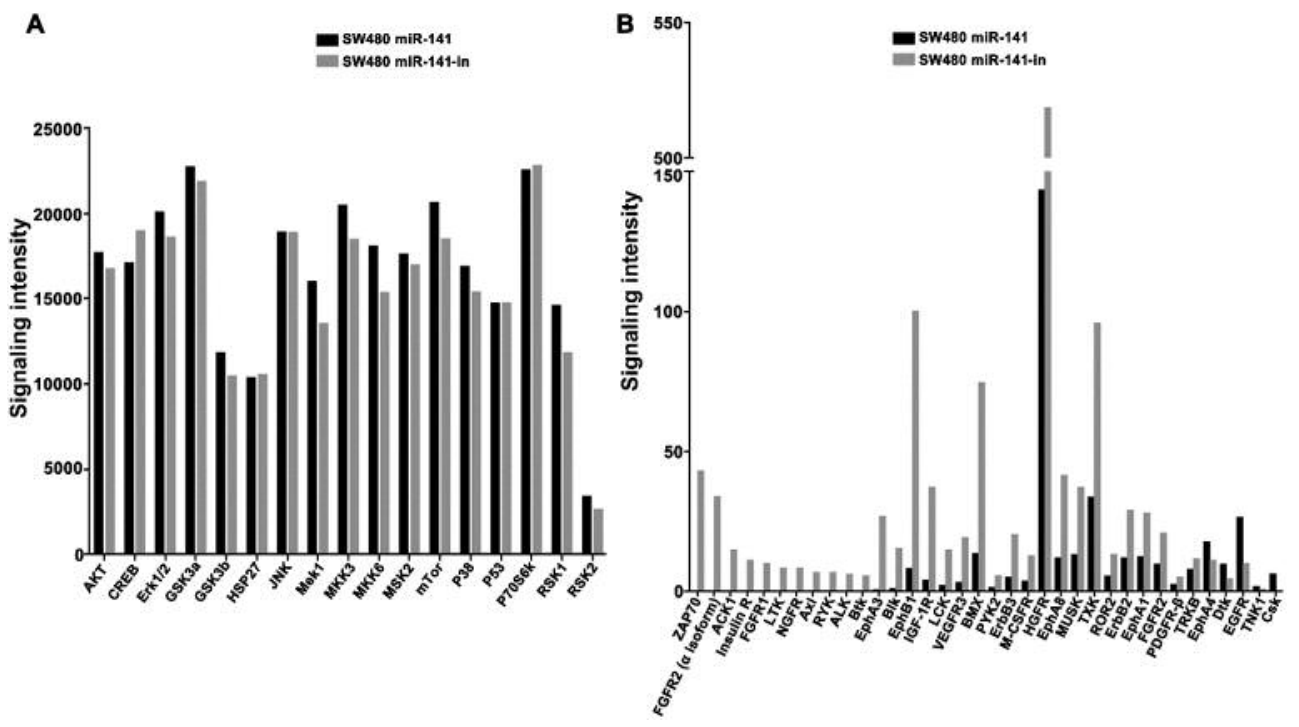

Figure 4. Results of human phospho-mitogen-activated protein kinase (MAPK) (A) and phospho-receptor tyrosine kinase, $(R T K)(B)$ arrays in miR141-overexpressing (miR-141) and-inhibited (miR-141-in) cells. The average signaling intensity of the spots for the representative signal protein is shown. Data are expressed as background-normalized pixel intensity.

detected following the inhibition of $m i R-141$ (>1.5-fold compared to $m i R-141$ cells, Figure 4B). In addition to highly activated HGFR, several other RTKs such as erythropoietin producing hepatocellular carcinoma B1 (EPHB1), insulinlike growth factor-1 receptor (IGF-1R), bone marrow kinase in chromosome X (BMX), and Txk tyrosine kinase (TXK) were also highly activated in SW480 cells when miR-141 was down-regulated. These RTKs were more activated in $m i R$ - 141 -in cells than in $m i R-141$ cells. These results suggest that miR-141 inhibits the activation of specific RTKs, such as HGFR.

\section{Discussion}

In the present study, we investigated the functional roles of miR-141 in CRC cell lines stably expressing $m i R-141$ and in cells with silencing of $m i R-141$. We found that $m i R-141$ overexpression significantly inhibited cell proliferation and migration of SW480 cells. miR-141 suppress cell-cycle progression by arresting cells in the $\mathrm{S}$ phase in our study. With overexpression of $m i R-141$, the proportion of cells in $\mathrm{G}_{1}$ phase increased and those in the $\mathrm{S}$ phase decreased. We also showed that $m i R-141$ suppressed the tumorigenicity and liver metastasis of SW480 cells in vivo. These results were similar to the findings of Feng et al. who showed that miR141 expression was down-regulated in CRC and influenced tumor progression in CRC metastases (8). Additionally, a study by $\mathrm{Hu}$ et al. also found that miR-141 inhibited the migration and invasion of SW620 CRC cells (9). All of these studies indicate that $m i R-141$ may act as a tumor suppressor and play an inhibitory role in the progression of CRC. However, our previous report revealed that high serum expression of $m i R-141$ contributed to liver metastasis in CRC (4). The reasons for these opposing findings may be that serum miRNAs are very stable (10), and $m i R-141$ leakage from damaged cells due to cancer could easily accumulate in blood to a high level.

Many studies indicated that dysregulation of $m i R-141$ depended on the type of cancer. $m i R-141$ may be a tumor suppressor gene or oncogene in different types of cancers, and modulate cellular migration through different mechanisms (11). Several studies revealed that miR-141 had multiple targets, among which were many oncogenes such as ZEB1/ZEB2, T-lymphoma invasion and metastasis 1 (TIAM1), hepatoma-derived growth factor (HDGF), and MAP4K4 (12-15). However, ZEB2 and MAP4K4 were confirmed as targets of $m i R-141$ in CRC $(8,9)$. In our study, we also found that down-regulation of $m i R-141$ in SW480 cells significantly increased the protein expression level of ZEB2. ZEB2 belongs to the zinc finger E-boxbinding homeobox family and is also known as SMADinteracting protein 1 (SIP1) (16). ZEB2 binds to the promoter of E-cadherin, leading to the down-regulation of E-cadherin expression, which is a hallmark of EMT (17). EMT is a progressive biological phenomenon whereby epithelial cells gradually acquire a mesenchymal (fibroblast-like) cell phenotype, and plays an important role in invasion and migration of cancer cells (18). Therefore, 
the present study hypothesized that in CRC cells, downregulation of $m i R-141$ might target the E-cadherin transcriptional repressor ZEB2 and promote EMT, cancer cell migration, and invasion. However, further studies are required to confirm this.

As in most other malignant tumor types, the migration and invasion of CRC is a very complicated biological processes involving multiple signaling pathways and various mechanisms. In order to find novel signaling pathways which could be targeted by $m i R-141$ in CRC cells, we used MAPK array and RTK array to detect which signal proteins were dysregulated in $m i R-141$ overexpressing and -down-regulated cells. The results of the present study indicated that $m i R-141$ does not affect the phosphorylation of 17 proteins of the MAPK pathway, including P53, ERK1/2, JNK, and P38. HGFR was the single most activated RTK, and we also detected other activated RTKs including IGF-1R, EPHB1, BMX and TXK in SW480 cells with down-regulation of $m i R-141$. HGFR is an RTK involved in cellular proliferation and apoptosis. It is overexpressed in the vast majority of invasive carcinomas and metastases in CRC (19). Hyperactivation of HGFR is related to malignant progression in different cancer types, including cell survival, growth, angiogenesis, cell motility, EMT, invasiveness and metastasis (20). Similarly, the IGF-1R is commonly overexpressed and activated in CRC and plays a critical role in proliferation, differentiation, migration, angiogenesis, and apoptosis of CRC cells (21). Cooperation between HGFR and IGF-1R was revealed in CRC cells, in which urokinase plasminogen activator (uPA) and its receptor (UPAR) is activated to regulate migration and invasion (22). Thus, we propose that the HGFR gene may be a target gene of $m i R-141$ in SW480 cells. Our future studies will focus on the $H G F R$ igene.

In conclusion, our study showed that $m i R-141$ appears to function as a tumor suppressor in CRC. Our results also identified a new regulatory network of the miR-141 HGFR nexus, which has not been reported in CRC development. Our research provides some insights into the functions of $m i R-141$.

\section{Acknowledgements}

This work was supported by the National Natural Science Foundation of China (Grant no. 81541050 and 81172317), the Beijing Natural Science Foundation of China (Grant no. 7154191), the Beijing Talent Training Funding of China (Grant no. 2014000021469G266 and 2016000021469G225), the Beijing Municipal Administration of Hospitals Clinical Medicine Development of Special Funding Support (Code: ZYLX201504), and the Beijing Municipal Administration of Hospitals' Youth Programme (Grant no. QML20160105). Beijing Municipal Administration of Hospitals Clinical Medicine Development of Special Funding Support, code: ZYLX201504. National Key Technologies R\&D Program (No. 2015BAI13B09).

\section{References}

1 Chen WQ, Zheng RS, Zhang SW, Zeng HM, Zuo TT, Jia MM, $\mathrm{Xia} \mathrm{CF}$, Zou XN and He J: Report of Cancer Incidence and Mortality in China. China Cancer 01: 1-8, 2016.

2 Siegel RL, Miller KD, and Jemal A: Cancer statistics. CA Cancer J Clin 65: 5-29, 2015.

3 Manfredi S, Lepage C, Hatem C, Coatmeur O, Faivre J and Bouvier AM: Epidemiology and management of liver metastases from colorectal cancer. Ann Surg 244: 254-259, 2006.

4 Yin J, Bai Z, Song J, Yang Y, Wang J, Han W, Zhang J, Meng H, Ma X, Yang Y, Wang T, Li W, and Zhang Z: Differential expression of serum $m i R-126, m i R-141$ and $m i R-21$ as novel biomarkers for early detection of liver metastasis in colorectal cancer. Chin J Cancer Res 26: 95-103, 2014.

5 Zhang X, Li P, Rong M, He R, Hou X, Xie Y, and Chen G: MicroRNA-141 is a biomarker for progression of squamous cell carcinoma and adenocarcinoma of the lung: clinical analysis of 125 patients. Tohoku J Exp Med 235: 161-169, 2015.

6 Okayama H, Schetter AJ and Harris CC: MicroRNAs and inflammation in the pathogenesis and progression of colon cancer. Dig Dis 2: 2:9-15, 2012.

7 Hur K: MicroRNAs: promising biomarkers for diagnosis and therapeutic targets in human colorectal cancer metastasis. BMB Rep 48: 217-222, 2015.

8 Feng L, Ma H, Chang L, Zhou X, Wang N, Zhao L, Zuo J, Wang Y, Han J and Wang G: Role of microRNA-141 in colorectal cancer with lymph node metastasis. Exp Ther Med 12: 3405$3410,2016$.

$9 \mathrm{Hu} \mathrm{M}$, Xia M, Chen X, Lin Z, Xu Y, Ma Y and Su L: MicroRNA-141 regulates SMAD-interacting protein 1 (SIP1) and inhibits migration and invasion of colorectal cancer cells. Dig Dis Sci 55: 2365-2372, 2010.

10 Mitchell PS, Parkin RK, Kroh EM, Fritz BR, Wyman SK, Pogosova-Agadjanyan EL, Peterson A, Noteboom J, O'Briant KC, Allen A, Lin DW, Urban N, Drescher CW, Knudsen BS, Stirewalt DL, Gentleman R, Vessella RL, Nelson PS, Martin DB and Tewari M: Circulating microRNAs as stable blood-based markers for cancer detection. Proc Natl Acad Sci USA 105: 10513-10518, 2008.

11 Bracken CP, Gregory PA, Kolesnikoff N, Bert AG, Wang J, Shannon MF and Goodall GJ: A double-negative feedback loop between ZEB1-SIP1 and the microRNA-200 family regulates epithelial-mesenchymal transition. Cancer Res 68: 7846-7854, 2008.

$12 \mathrm{Xu} \mathrm{H}$, Mei Q, Xiong C and Zhao J: Tumor-suppressing effects of miR-141 in human osteosarcoma. Cell Biochem Biophys 69: 319-325, 2014

13 Liu Y, Ding Y, Huang J, Wang S, Ni W, Guan J, Li Q, Zhang Y, Ding Y, Chen B and Chen L: miR-141 suppresses the migration and invasion of HCC cells by targeting TIAM1. PLoS One 9: e88393, 2014.

14 Chen B, Huang T, Jiang J, Lv L, Li H and Xia S: miR-141 suppresses proliferation and motility of gastric cancer cells by targeting HDGF. Mol Cell Biochem 388: 211-218, 2014.

15 Zhao G, Wang B, Liu Y, Zhang JG, Deng SC, Qin Q, Tian K, Li $\mathrm{X}$, Zhu S, Niu Y, Gong Q and Wang CY: miRNA-141, downregulated in pancreatic cancer, inhibits cell proliferation and invasion by directly targeting MAP4K4. Mol Cancer Ther 12: 2569-2580, 2013. 
16 Sekido R, Murai K, Funahashi J, Kamachi Y, Fujisawa-Sehara A, Nabeshima Y and Kondoh H: The delta-crystallin enhancerbinding protein delta EF1 is a repressor of E2-box-mediated gene activation. Mol Cell Biol 14: 5692-5700, 1994.

17 Vandewalle C, Comijn J, De Craene B, Vermassen P, Bruyneel E, Andersen H, Tulchinsky E, Van Roy F and Berx G: SIP1/ZEB2 induces EMT by repressing genes of different epithelial cell-cell junctions. Nucleic Acids Res 33: 6566-6578, 2005.

18 Kraljevic Pavelic S, Sedic M, Bosnjak H, Spaventi S and Pavelic $\mathrm{K}$ : Metastasis: new perspectives on an old problem. Mol Cancer 10: 22, 2011.

19 Di Renzo MF, Olivero M, Giacomini A, Porte H, Chastre E, Mirossay L, Nordlinger B, Bretti S, Bottardi S and Giordano S: Overexpression and amplification of the MET/HGF receptor gene during the progression of colorectal cancer. Clin Cancer Res 1: 147-154, 1995.

20 Yap TA, Sandhu SK, Alam SM and de Bono JS: HGF/c-MET targeted therapeutics: novel strategies for cancer medicine. Curr Drug Targets 12: 2045-2058, 2011.
21 Inno A, Di Salvatore M, Cenci T, Martini M, Orlandi A, Strippoli A, Ferrara AM, Bagalà C, Cassano A, Larocca LM and Barone C: Is there a role for IGF1R and c-MET pathways in resistance to cetuximab in metastatic colorectal cancer? Clin Colorectal Cancer 10: 325-332, 2011.

22 Bauer TW, Fan F, Liu W, Johnson M, Parikh NU, Parry GC, Callahan J, Mazar AP, Gallick GE and Ellis LM: Insulin-like growth factor-I-mediated migration and invasion of human colon carcinoma cells requires activation of c-MET and urokinase plasminogen activator receptor. Ann Surg 241: 748-758, 2005.

Received May 4, 2017

Revised June 9, 2017

Accepted June 13, 2017 